\title{
Photoluminescence and thermoluminescence properties of tricalcium phosphate phosphors doped with dysprosium and europium
}

\author{
K MADHUKUMAR*, H K VARMA ${ }^{\dagger}$, MANOJ KOMATH ${ }^{\dagger}$, T S ELIAS ${ }^{\ddagger}$, \\ V PADMANABHAN ${ }^{\ddagger}$ and C M K NAIR \\ Department of Physics, Mahatma Gandhi College, Thiruvananthapuram 695 004, India \\ ${ }^{\dagger}$ Sree Chitra Tirunal Institute for Medical Sciences and Technology, Thiruvananthapuram 695012 , India \\ ${ }^{\ddagger}$ Regional Cancer Centre, Thiruvananthapuram 695 011, India
}

MS received 8 March 2007; revised 18 July 2007

\begin{abstract}
The suitability of calcium phosphate crystals for thermoluminescence dosimetry (TLD) applications is investigated, owing to their equivalence to bone mineral. The $\alpha$ and $\beta$ phases of tricalcium phosphate (TCP) were synthesized through wet precipitation and high temperature solid state routes and doped with Dy and Eu. The photoluminescence and thermoluminescence studies of the phosphors have been carried out.

The TL properties were found to be highly dependent on the method of preparation of the material. Eu doping gave good PL emission, whereas Dy doping was more efficient in TL emission. $\beta$-TCP was found to be less TL sensitive than $\alpha$-TCP, yet it was identified as a better phosphor material owing to its better fading characteristics.

The dependence of TL of $\beta$-TCP : Dy on the energy and dose of radiation, and on the doping concentration were studied. The TL intensity was observed to fade exponentially during a storage period of 20 days, but it stabilized at $70 \%$ of the initial value after 30 days. The optimum doping concentration was found to be $0.5 \mathrm{~mol} \%$.
\end{abstract}

Keywords. Photoluminescence; thermoluminescence; radiation dosimetry.

\section{Introduction}

Phosphors owe their practical importance to their property of absorbing incident energy and converting it into visible radiations. This phenomenon, known as luminescence, is driven by electronic processes in the material due to the presence of trapping levels created by the presence of impurity atoms or lattice defects. The study of absorption and emission characteristics of a phosphor enables the understanding of electronic energy levels in the crystal and, in turn, the design of new phosphors of practical relevance (McKinlay 1981; Mckeever 1985).

Thermoluminescence (TL) is a related property shown by phosphors. This phenomenon involves radiative recombination of thermally released electrons or holes from their traps, which were created by exposure to ionizing radiation. This is an efficient tool for studying the electronic trap levels in crystals, and finds practical application in thermoluminescence dosimetry (TLD) used for radiation dose measurements. TLD is based on the principle that the amount of light released by the phosphor material, which has been previously exposed to ionizing radia-

\footnotetext{
*Author for correspondence (madhusandh@yahoo.co.in)
}

tion, will depend on the radiation dose received by the material. TLD badges, which carry specially tuned phosphor material, are widely used in personnel as well as environmental radiation monitoring. Though a large number of organic and inorganic materials exhibit thermoluminescence, only a small fraction possesses all the ideal characteristics of a good TLD phosphor (McKinlay 1981; Mckeever 1985).

Most widely used and extensively studied materials for TLD are sulfates, sulfides and fluorides of alkali and alkaline earth elements. Phosphates and halo-phosphates of alkali and alkaline earth elements, used for luminescence applications, were also explored for their suitability as TLD materials (Welker 1991; Band et al 1997; Kottaiswamy et al 1997; Sanayee et al 1997; Dhabekar et al 2002). Schipper et al (1993) studied trapping of electrons in the X-ray storage phosphor, $\mathrm{Ba}_{3}\left(\mathrm{PO}_{4}\right)_{2}: \mathrm{Eu}, \mathrm{La}$. Seshagiri et al (1992) studied the thermally stimulated luminescence (TSL) and electron paramagnetic resonance (EPR) properties of calcium chlorophosphates. The possible mechanism of TSL glow is proposed by correlating the spectral characteristics and the thermal stability of the radical ions. TL of mixed rare earth phosphate powder was studied by Michel et al (2001), who observed that the TL emission spectra of the phosphor mainly composed of characteristic line transition of rare earth ions. 
Calcium phosphate crystals also are candidate materials for TLD applications (Ohtaki et al 1993). This interest stems out of their equivalence to bone mineral. Calcium phosphates (especially tricalcium phosphate, calcium hydroxyapatite and their combinations) are already in clinical use as ceramic bone substitutes, by virtue of their osteoconductive nature (Kanazawa 1989). The dosimetry aspects of calcium phosphates have special significance in the wake of widespread use of X-rays in the diagnostic and therapeutic applications.

A series of calcium phosphate compounds is available: dicalcium phosphate $\left[\mathrm{CaHPO}_{4}\right]$, tricalcium phosphate $\left[\mathrm{Ca}_{3}\left(\mathrm{PO}_{4}\right)_{2}\right]$, tetracalcium phosphate $\left[\mathrm{Ca}_{4}\left(\mathrm{PO}_{4}\right)_{2} \mathrm{O}\right]$ and hydroxyapatite $\left[\mathrm{Ca}_{10}\left(\mathrm{PO}_{4}\right)_{6}(\mathrm{OH})_{2}\right]$, which differ by their chemical stoichiometry and crystallographic structure. The defining factor is $\mathrm{Ca} / \mathrm{P}$ ratio which lies between 1 and 2. Among these, tricalcium phosphate, with a $\mathrm{Ca} / \mathrm{P}$ ratio $1 \cdot 5$, seemed to be a good candidate for TLD applications by virtue of its crystal structure. Four polytypes of TCP are known: $\beta$-TCP, $\alpha$-TCP, $\alpha^{\prime}$-TCP and a highpressure form of $\gamma$-TCP (Jarcho et al 1976; Ducheyne and de Groot 1981; Kanazawa 1989).

In this paper, an investigation of the possibility of using $\alpha$ and $\beta$ phases of tricalcium phosphates for TLD phosphor application, is taken up. This work is stimulated by the studies of Fukuda et al (1992). The workers studied TCP doped with Dy, Tm, Sm and Ce and suggested that the material may be useful for dosimetry applications (Fukuda et al 1992, 1993; Mizuguchi and Fukuda 1999).

The excitation and emission characteristics, as well as the biological, chemical, crystallographic and physical properties of calcium phosphates are greatly influenced by the method of preparation (Jarcho et al 1976; Ducheyne and de Groot 1981; Kanazawa 1989). Therefore, in the present work, $\alpha$ and $\beta$ phases of tricalcium phosphate (TCP) doped with rare earth ions, dysprosium (Dy) and europium (Eu), were prepared through two different routes and their luminescence properties investigated. The details of preparation methods and studies on the luminescence characteristics of the materials are given in the following sections.

\section{Experimental}

The initial part of the work was the preparation of doped phosphors. Tricalcium phosphate (TCP) crystals, in both $\alpha$ and $\beta$ phases, were synthesized through two different routes, viz. wet precipitation and high temperature solid state reaction. The doping was done during the synthesis using suitable compounds of dysprosium and europium. All chemicals used in the study were of AR grade.

To investigate the characteristic emissions of dysprosium and europium, $\beta$-TCP phase was doped with $0.1 \mathrm{~mol} \%$ of these dopants and the excitation and emission spectra were taken. Thereafter, the thermoluminescence (TL) characteristics of $\alpha$-TCP and $\beta$-TCP, doped with Dy and
Eu, were studied. The doped TCP phosphor materials processed at different annealing conditions were subjected to TL studies, to observe the effects of phase change. The TL responses of dysprosium doped $\beta$-TCP samples prepared through wet precipitation as well as solid state synthesis were compared.

Subsequently, the suitability of Dy doped $\beta$-TCP phosphor for TLD applications was explored. The energy sensitivity of $\beta$-TCP : Dy phosphor was recorded after subjecting to X-ray energies. Also, the dose dependence of the same was studied after irradiating with different known doses of X-rays. Periodic TL retention of the phosphors, irradiated with a known dose of X-rays and stored in ambient conditions, was analysed to see fading. Efforts were made to determine the optimum dopant concentration by analysing the TL sensitivity of $\beta$-TCP phosphors doped with varying concentrations of dysprosium.

\subsection{Material preparation}

2.1a Wet precipitation method: The wet reaction is carried out using calcium nitrate and diammonium hydrogen phosphate in an ammoniated solution. The precipitation of tricalcium phosphate occurs through the chemical reaction

$3 \mathrm{Ca}\left(\mathrm{NO}_{3}\right)_{2}+2\left(\mathrm{NH}_{4}\right)_{2} \mathrm{HPO}_{4}+2 \mathrm{NH}_{4} \mathrm{OH} \rightarrow \mathrm{Ca}_{3}\left(\mathrm{PO}_{4}\right)_{2}$

$+6 \mathrm{NH}_{4} \mathrm{NO}_{3}+2 \mathrm{H}_{2} \mathrm{O}$.

The doping was done by adding oxides of the dopant elements (dysprosium and europium) dissolved in minimum quantity of dilute nitric acid.

$500 \mathrm{ml}$ of $0.6 \mathrm{M}$ calcium nitrate solution was prepared in a 21 beaker using distilled water. The selected dopant compound (dissolved in nitric acid) was added in appropriate quantities to the solution. The contents were well stirred using a magnetic stirrer. $500 \mathrm{ml}$ of $0.4 \mathrm{M}$ diammonium hydrogen phosphate was prepared in another beaker and the $\mathrm{pH}$ was adjusted to 10 by adding ammonia solution. The solution was taken in a dropping funnel and allowed to fall drop by drop to the beaker containing calcium nitrate solution under constant stirring. White precipitate of calcium phosphate was formed. Stirring was continued for $30 \mathrm{~min}$ more, maintaining the $\mathrm{pH}$ at 10 .

The content in the beaker was kept undisturbed for $24 \mathrm{~h}$ for maturation, and thereafter, the supernatant liquid was decanted to collect the precipitate. It was then centrifuged thrice using distilled water and finely filtered. The filtrate was dried at $100^{\circ} \mathrm{C}$ in a hot air oven overnight and then calcined at $300^{\circ} \mathrm{C}$ in a muffle furnace for $3 \mathrm{~h}$ to remove any traces of other compounds.

The calcined material was ground to form fine powder and graded using standard sieves. It was then sintered at high temperatures for $2 \mathrm{~h}$ in a programmable furnace to obtain the required phase $\left(900^{\circ} \mathrm{C}\right.$ for $\beta$-TCP and $1300^{\circ} \mathrm{C}$ for $\alpha$-TCP). Various samples were prepared using dysprosium and europium as dopants. 
2.1b High temperature solid state synthesis: The solid state synthesis of tricalcium phosphate was done through a high temperature firing of the powder mixture of calcium oxide $(\mathrm{CaO})$ and dicalcium phosphate $\left(\mathrm{CaHPO}_{4}\right)$. The reaction governing the process is

$$
\mathrm{CaO}+2 \mathrm{CaHPO}_{4} \rightarrow \mathrm{Ca}_{3}\left(\mathrm{PO}_{4}\right)_{2}+\mathrm{H}_{2} \mathrm{O} .
$$

$\mathrm{CaO}$ and $\mathrm{CaHPO}_{4}$ powders were taken in $1: 2$ molar ratio and mixed thoroughly using mortar and pestle with acetone as the medium. The dopant, in powder form, in appropriate amount was added to this, again mixed thoroughly for $1 \mathrm{~h}$, and then transferred to a porcelain crucible. The powder was heated at about $300^{\circ} \mathrm{C}$, then mixed for $1 \mathrm{~h}$, and annealed at $1100^{\circ} \mathrm{C}$ for $2 \mathrm{~h}$. The resulting compound was crushed to powder from and graded using standard sieves.

\subsection{Sample characterization}

The samples prepared through different routes were subjected to X-ray diffraction analysis. Spectrogram was obtained using Siemens D5005 XRD. $\mathrm{CuK}_{\alpha}$ radiation was used for collecting the spectra.

\subsection{Luminescence studies}

PL studies were carried out using a Hitachi Fluorescence spectrophotometer, model F-4000. The excitation/emission spectra were analysed to study the nature of emission centres and the type of emission.

TL studies were carried out using a PC based TL analyzer, type TL1007 (NUCLEONIX). $10 \mathrm{mg}$ of powder samples with grain size around $200 \mu \mathrm{m}$ were used for TL measurements. RADON make X-ray unit $(30 \mathrm{kV}, 10 \mathrm{~mA})$ was used for irradiating the sample.

\section{Results and discussion}

\subsection{X-ray diffraction analysis}

Figure 1 shows the typical XRD patterns of $\alpha$-TCP and $\beta$-TCP samples obtained after synthesis. The samples prepared by wet precipitation (annealed at $900^{\circ} \mathrm{C}$ ) and by solid state sintering (annealed at $1100^{\circ} \mathrm{C}$ ) techniques gave the spectra which match perfectly with that of $\beta$-TCP (Whitlockite mineral phase, JCPDS File No. 09-0169). The XRD pattern of sample prepared by wet precipitation

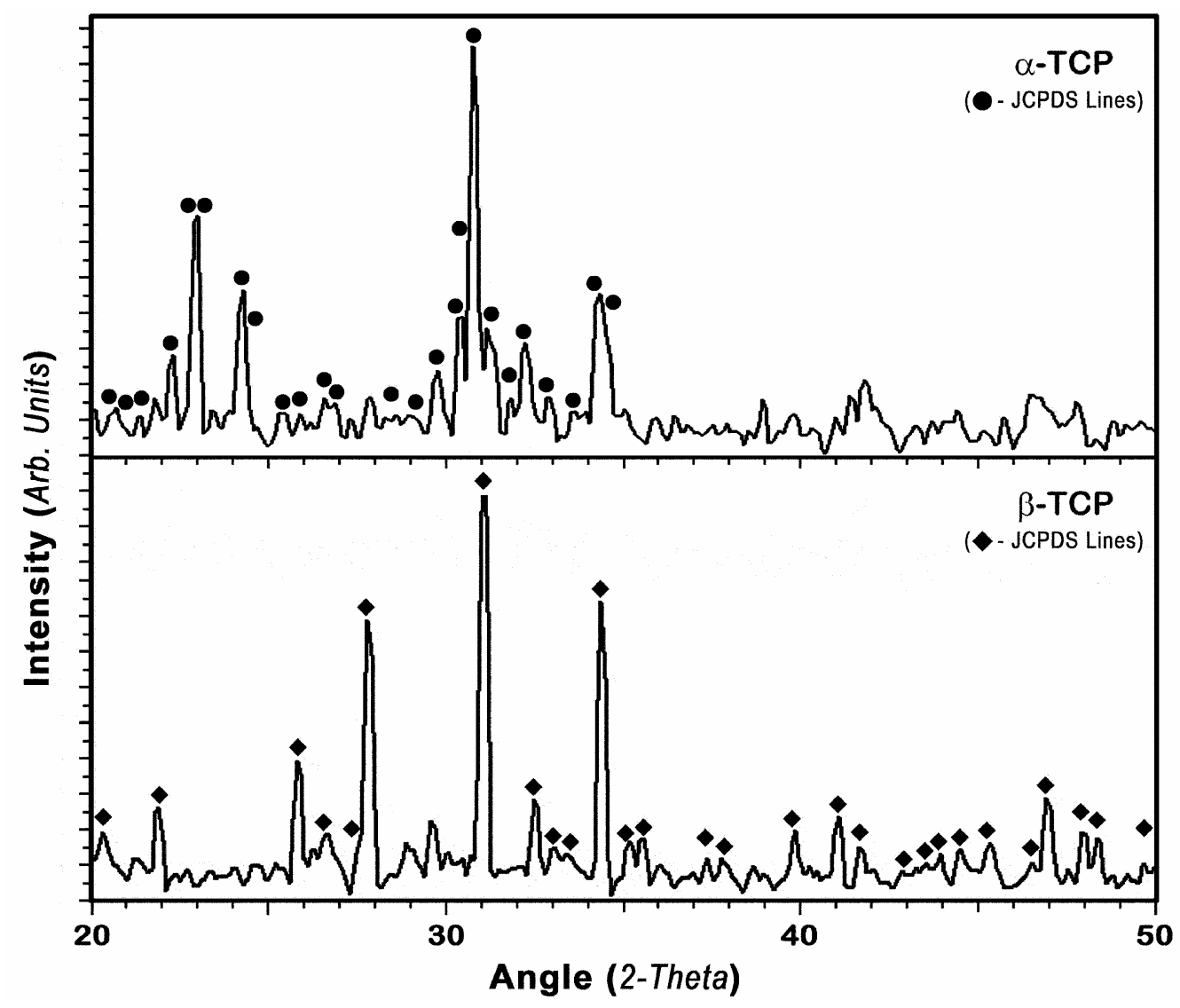

Figure 1. Typical XRD spectra of $\alpha$-TCP and $\beta$-TCP. The standard JCPDS lines corresponding to each compound are marked. 

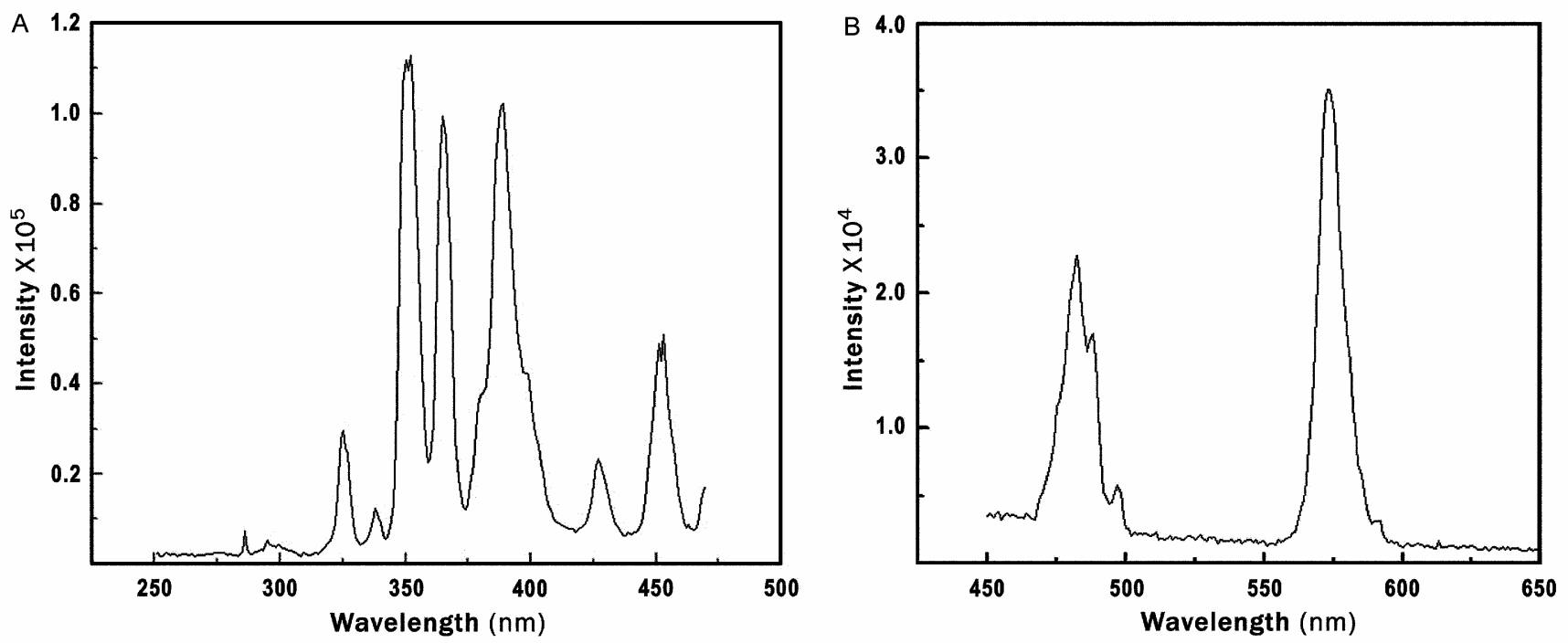

Figure 2. Photoluminescence spectra of $\beta$-TCP phosphor doped with $0 \cdot 1 \%$ Dy. The spectrum A shows the excitation at the emission wavelength of $580 \mathrm{~nm}$, and spectrum B, the emission at an excitation wavelength of $351 \mathrm{~nm}$.
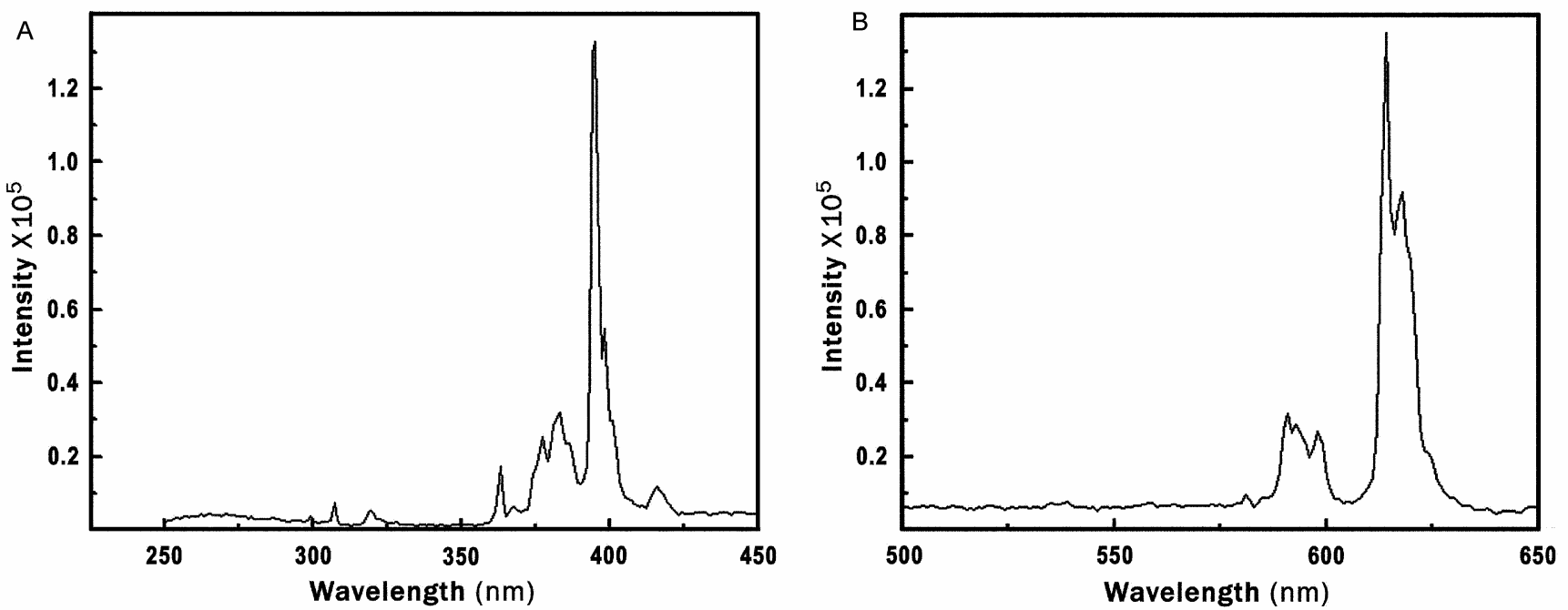

Figure 3. Photoluminescence spectra of $\beta$-TCP phosphor doped with $0 \cdot 1 \%$ Eu. The spectrum A shows the excitation at the emission wavelength of $613 \mathrm{~nm}$, and spectrum B, the emission at an excitation wavelength of $394 \mathrm{~nm}$.

method and annealed at $1300^{\circ} \mathrm{C}$ matched with that of $\alpha$-TCP (JCPDS file number 09-0348).

\subsection{Photoluminescence studies}

3.2a Dysprosium-doped TCP: Excitation spectrum of $\beta$-TCP doped with $0.1 \mathrm{~mol} \%$ Dy (figure $2 \mathrm{~A}$ ) consists of a most intense peak at $351 \mathrm{~nm}$ and two slightly lesser in tense peaks at $364 \mathrm{~nm}$ and $388 \mathrm{~nm}$, respectively when scanned for an emission wavelength, $\lambda_{\mathrm{em}}=580 \mathrm{~nm}$. Weak peaks are also observed in the region $350-450 \mathrm{~nm}$. The optical absorption transitions corresponding to these wavelengths are within the $4 f$ shell.
Figure 2B shows the PL emission spectrum of $\beta$ TCP : Dy $(0 \cdot 1 \mathrm{~mol} \%)$ for an excitation wavelength, $\lambda_{\mathrm{ex}}=$ $351 \mathrm{~nm}$. Characteristic emission peaks observed at 482 and $572 \mathrm{~nm}$ are corresponding to transitions ${ }^{4} F_{9 / 2} \rightarrow{ }^{6} H_{15 / 2}$ and ${ }^{4} F_{9 / 2} \rightarrow{ }^{6} H_{13 / 2}$. This is a clear indication of $\mathrm{Dy}^{3+}$ ion acting as luminescence centre (Nakashima et al 2005).

3.2b Europium doped TCP: The excitation spectrum of $\beta$-TCP : Eu $(0 \cdot 1 \mathrm{~mol} \%)$, shown in figure $3 \mathrm{~A}$ has an intense peak around $394 \mathrm{~nm}\left(\lambda_{\mathrm{em}}=613 \mathrm{~nm}\right)$. Weak peaks are also observed in the region between 300 and $500 \mathrm{~nm}$. The optical absorption transition corresponding to this wavelength is within the $4 f$ shell. The peak observed at 
$394 \mathrm{~nm}$ corresponds to ${ }^{7} F_{0} \rightarrow{ }^{5} L_{6}$ transition of $\mathrm{Eu}^{3+}$ ions.

The emission spectrum (figure 3B) has a strong emission peak at $613 \mathrm{~nm}$ corresponding to the transition, ${ }^{5} D_{0} \rightarrow{ }^{7} F_{2}$. Weak band around $591 \mathrm{~nm}$ corresponds to ${ }^{5} D_{0} \rightarrow{ }^{7} F_{1}$ transitions. The strong emission peak observed at $613 \mathrm{~nm}$ indicates that the impurity ion is located mostly at a site other than the centre of symmetry of the crystal lattice. The multiple emission lines seen at each transition are due to the crystal field splitting of the ground state of the emitting ions (Doata et al 2005). Weak emission corresponding to ${ }^{6} P_{7 / 2} \rightarrow{ }^{6} S_{7 / 2}$ transitions of $\mathrm{Eu}^{2+}$ was also observed in the sample (spectrum not included).

\subsection{Thermoluminescence studies}

No TL emission was observed in undoped TCP samples when irradiated with X-rays. The TL response of $\alpha$-TCP and $\beta$-TCP, the two major phases of TCP, differed much in their glow peak patterns and sensitivity.

Figure 4 shows a comparison of TL response of $\alpha$-TCP and $\beta$-TCP doped with $0 \cdot 1 \mathrm{~mol} \%$ Dy (wet precipitation) and irradiated with $30 \mathrm{kV}, 10 \mathrm{~mA}$ X-ray beam. An intense glow peak is seen at $118^{\circ} \mathrm{C}$ for $\alpha$-TCP whereas multipeaks at 140,215 and $273^{\circ} \mathrm{C}$ are seen for $\beta$-TCP, when the same heating rate was applied for both the samples. The peak at $118^{\circ} \mathrm{C}$ for $\alpha$-TCP is nearly twice as intense as the most intense peak observed at $273^{\circ} \mathrm{C}$ for $\beta$-TCP (wet precipitation).

Though $\alpha$-TCP appears to be much more TL sensitive than $\beta$-TCP, it may not be suitable for dosimetric applications, as the low temperature peak is an indication of high fading rate (Mckeever 1985). The observed shift in the glow peak position towards low temperature side in $\alpha$ TCP may be due to the change of phase in the crystal structure as seen in their XRD patterns (figure 1).

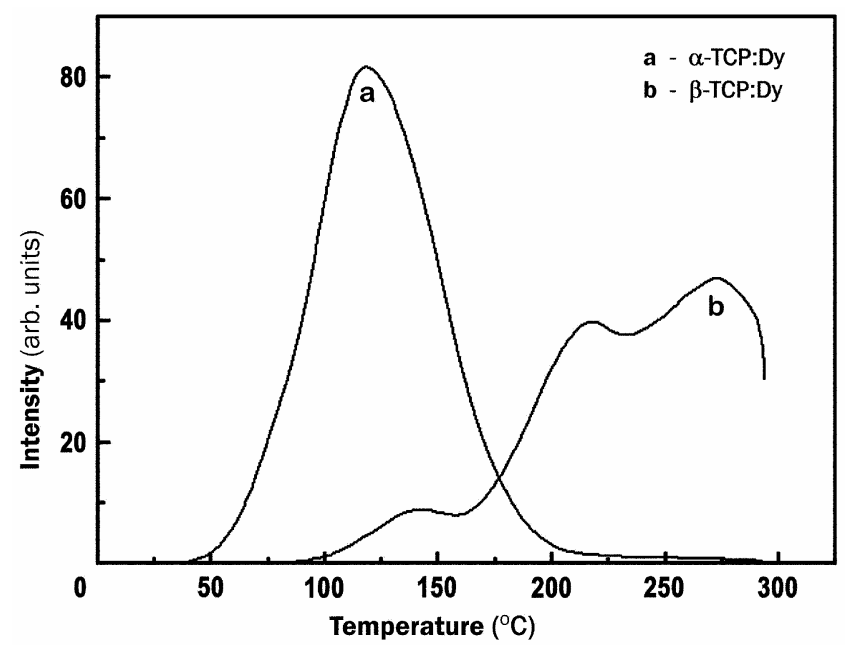

Figure 4. TL glow curves of $\alpha$-TCP and $\beta$-TCP doped with $0 \cdot 1 \%$ Dy.
The TL glow curves of $\beta$-TCP:Dy and $\beta$-TCP:Eu prepared by solid state synthesis under the same conditions are shown in figure 5 . It is seen that the TL response of $\beta$-TCP:Eu is very low when compared to that of $\beta$-TCP : Dy. Therefore, for further TL studies, $\beta$-TCP doped with Dy alone is used.

Eu doped $\beta$-TCP shows high PL emission (3 times compared to Dy doping, as seen in figure 3B) and low TL emission. This may be due to poor energy transfer from the luminescence centre to the activator ions. The TL mechanism of $\beta$-TCP : Dy could be explained as follows. The trapping and recombination seems to be characteristic of host lattice. During irradiation, the $\mathrm{Dy}^{3+}$ ions may change its valence state to $\mathrm{Dy}^{2+}$ by trapping an electron. The holes released simultaneously might also be trapped by the $\mathrm{Ca}_{3}\left(\mathrm{PO}_{4}\right)_{2}$ matrix forming radicals derived from $\mathrm{PO}_{4}^{3-}$. Some of these defects recombine immediately after creation while other pairs separate by migration and remain as stable defects. The formation and stability of defects centres depend on the method of preparation of the phosphor. During heating the trapped electrons and/or holes will be released and recombine with each other leading to TL of $\mathrm{Dy}^{3+}$.

3.3a Effect of annealing temperature on TL: The effect of annealing temperature and annealing duration on the TL response of Dy doped $\beta$-TCP was studied in detail. It was observed that both these parameters influence the TL response of $\beta$-TCP phosphors to a great extent. Figure 6 shows TL response of $\beta$-TCP:Dy phosphor prepared through wet precipitation followed by annealing at 900 and $1000^{\circ} \mathrm{C}$, with 2 time periods each. All the samples had been uniformly irradiated with X-rays prior to TL analysis. It is observed that the intensity of high temperature peaks at 273 and $215^{\circ} \mathrm{C}$ in the sample A (annealed at $900^{\circ} \mathrm{C} / 2 \mathrm{~h}$ ) decreases with an increase in annealing tem-

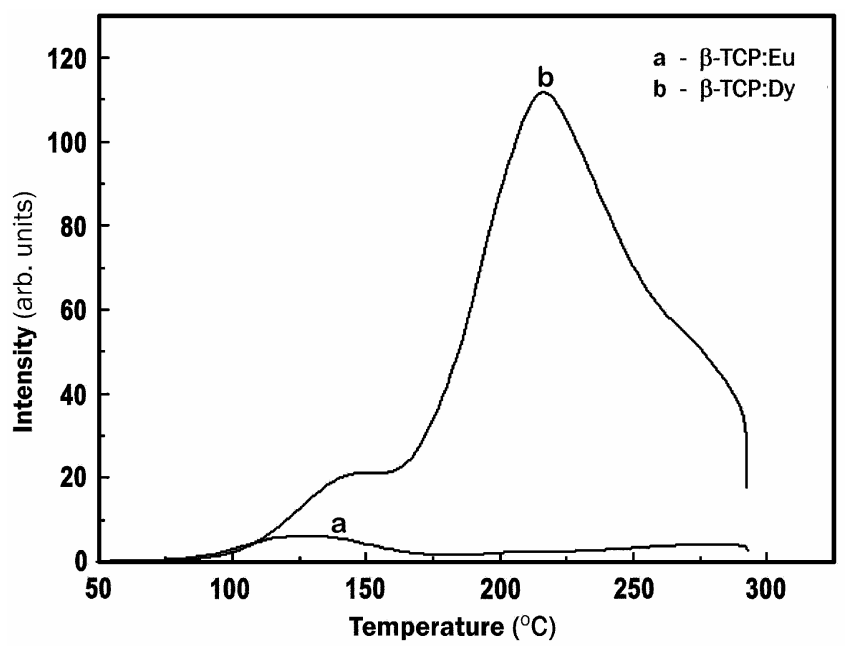

Figure 5. TL glow curves of $\beta$-TCP : Eu and $\beta$-TCP : Dy prepared by solid state synthesis, doped at $0 \cdot 1 \mathrm{~mol} \%$ each. 
perature and annealing duration while the intensity of low temperature peak at $140^{\circ} \mathrm{C}$ is found to increase. A shift in the peak temperature towards lower side is seen as the annealing temperature and duration is increased.

The above result seems very interesting. In figure 6 , for $900^{\circ} \mathrm{C}$ and $2 \mathrm{~h}$ annealing, peaks are observed at $140^{\circ} \mathrm{C}$, $215^{\circ} \mathrm{C}$ and $273^{\circ} \mathrm{C}$. The peak at $140^{\circ} \mathrm{C}$ is weak compared to those at $215^{\circ} \mathrm{C}$ and $273^{\circ} \mathrm{C}$. When the annealing duration is doubled for the same temperature (sample b), the high temperature peak heights at $273^{\circ} \mathrm{C}$ and $215^{\circ} \mathrm{C}$ are reduced considerably while there is nearly a seven-fold increase in the intensity of peak at $140^{\circ} \mathrm{C}$. This decrease in intensity of high temperature peaks and increase in intensity of low temperature peak continues with increase in annealing temperature and duration until the high temperature peaks disappear and low temperature peak alone exists with maximum intensity (samples $c$ and $d$ ). It is notable that the curve of sample $d$ exactly fits in with that of $\alpha$-TCP, which is a clear indication of the change of phase from $\beta$ to $\alpha$.

This result brings out the fact that thermoluminescence (TL) is an efficient and dependable tool for identification of phase changes with temperature.

$3.3 \mathrm{~b}$ Comparison of TL response of $\beta$-TCP phosphors prepared by different routes: The TL response of $\beta$-TCP samples prepared through wet precipitation as well as solid state synthesis was studied. Figure 7 shows the glow curves of $\beta$-TCP : Dy $(0 \cdot 1 \mathrm{~mol} \%)$ phosphors prepared by wet precipitation and solid state synthesis. Annealing temperatures, $900^{\circ} \mathrm{C} / 2 \mathrm{~h}$ and $1100^{\circ} \mathrm{C} / 2 \mathrm{~h}$, were optimized for pure phase formation ( $\beta$-TCP) in wet precipitation method and solid state synthesis, respectively. The TL response was studied after irradiating the samples with same dose of X-rays. Same heating rate was applied dur-

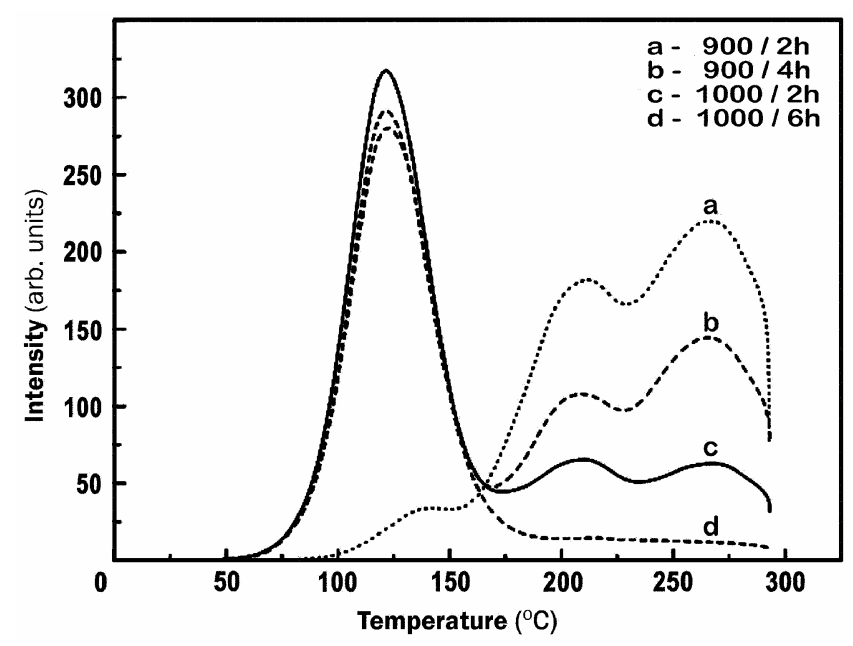

Figure 6. Glow curves of $\beta$-TCP samples doped with $0 \cdot 1 \%$ Dy annealed at different temperatures and periods, with a heating rate of $4^{\circ} \mathrm{C} / \mathrm{s}$. ing TL readout. It is seen that the phosphor prepared through wet precipitation shows peaks at 140, 215 and $273^{\circ} \mathrm{C}$ (intense), whereas the solid state synthesized sample has a prominent peak at $220^{\circ} \mathrm{C}$ with shoulders at $140^{\circ} \mathrm{C}$ and $273^{\circ} \mathrm{C}$. The ratio of intensity of peak height at 140 and $220^{\circ} \mathrm{C}$ is nearly 5 , which is a clear indication that the $220^{\circ} \mathrm{C}$ peak is almost an isolated one which can be used for radiation dosimetry applications. The phosphor prepared through wet precipitation is more TL sensitive than that prepared by solid state synthesis. The defect chemistry and particle sizes might have played a role in this effect. However, it cannot be recommended for dosimetry applications because of the presence of multi-peaks in the TL emission.

A well-defined high temperature peak is the foremost requirement of a good TL dosimeter. Hence, further TL analyses were performed only with samples prepared through solid state synthesis.

3.3c Variation of TL sensitivity with energy of radiation: The energy response of a dosimeter is an important parameter, which decides its suitability in gadgets for personnel monitoring. X-rays of different energies are being used in medical applications, with low energy for diagnostic applications and much higher energies in therapeutic applications. An ideal dosimeter should respond to all energies of radiation with same sensitivity.

To study the energy response of $\beta$-TCP phosphor, irradiation was carried out using a Elite-80 (Siemens model) Simulator unit. Samples were exposed to X-rays of various energies from 10-150 keV keeping the tube current and exposure time settings constant. The samples were kept at a fixed distance from the source and the actual exposures at this distance were measured with standard dosimeters.

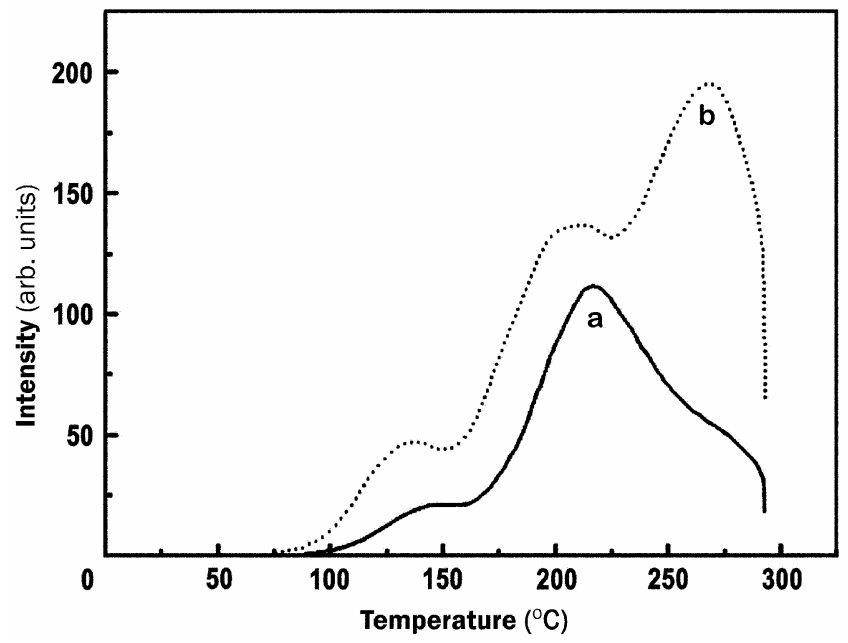

Figure 7. Glow curves of $\beta$-TCP prepared through different routes. The curve a denotes the solid state synthesis and $\mathbf{b}$, the wet precipitation. 
The energy response study (figure 8) shows a linear increase of TL response with the increase in X-ray energy. It reaches a maximum at around $50 \mathrm{keV}$ and then decreases steadily till $100 \mathrm{keV}$. Thereafter, it remains at nearly a constant value. This is due to the higher photoelectric absorption coefficient of the material for low energy radiations.

$3.3 \mathrm{~d}$ Dependence of TL on radiation dose: The dose dependence of TL emission of $\beta$-TCP : Dy phosphor was studied after irradiating the phosphor with different doses of X-rays at $50 \mathrm{keV}$ energy. Figure 9 shows the TL response of $220^{\circ} \mathrm{C}$ peak of $\beta$-TCP : Dy $(0.1 \mathrm{~mol} \%)$ with the dose. The intensity of $220^{\circ} \mathrm{C}$ peak increases linearly up to an exposure dose of $10 \mathrm{Ckg}^{-1}$ and saturates for higher doses.

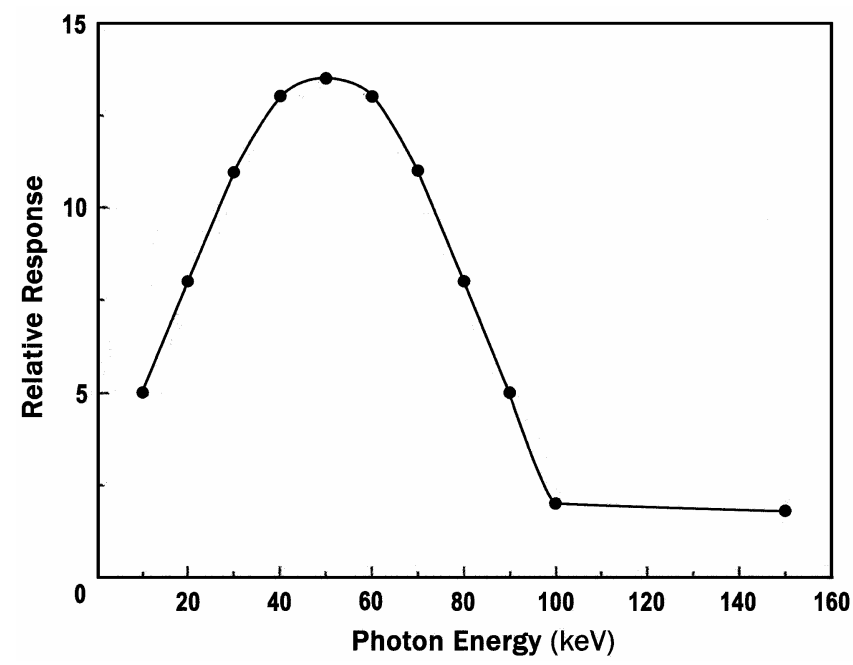

Figure 8. Energy response of $\beta$-TCP phosphor doped with $0 \cdot 1 \%$ Dy.

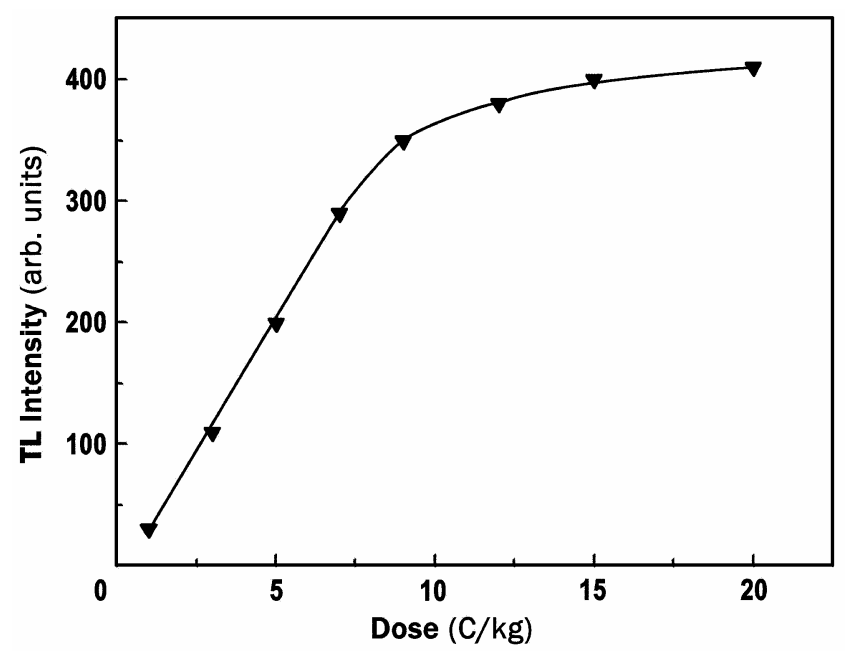

Figure 9. Dose response of $\beta$-TCP phosphor doped with $0 \cdot 1 \%$ Dy. 3.3e Fading: The fading characteristic of a phosphor indicates its ability to retain charge pairs created during irradiation over the storage period. The $\beta$-TCP:Dy $(0.1 \mathrm{~mol} \%)$ phosphor was irradiated at $10 \mathrm{Ckg}^{-1}$ dose of $\mathrm{X}$-rays and kept at room temperature. Periodic TL analysis was made at intervals of $24 \mathrm{~h}$, for a period of 30 days. Figure 10 shows the variation of TL response of $220^{\circ} \mathrm{C}$ peak. It is seen that an exponential fading occurs during a storage period of 20 days, but the value gets stabilized at $70 \%$ of the initial value after 30 days. According to accepted conventions (Pradhan 1997), the fading of a phosphor should be $<20 \%$ within 30 days at ambient temperatures up to $50^{\circ} \mathrm{C}$. In the present case, $20 \%$ fading occurs in the first 10 days of storage. Though this is on the higher side, the fading of $\beta$-TCP : Dy phosphors do not go below $30 \%$ of the initial value, even after 30 days.

3.3f Effect of dopant concentration on TL sensitivity: The optimum dopant concentration is an important factor in the designing of a TLD phosphor. The above studies of TL characteristics of Dy doped $\beta$-TCP samples were done at $0.1 \mathrm{~mol} \%$ doping level. It was interesting to look into the optimum concentration of doping for this particular combination.

TCP samples with varying Dy concentrations, from $0 \cdot 1-0.8 \mathrm{~mol} \%$, were prepared through solid state synthesis technique. Figure 11 shows the variation of TL intensity of $220^{\circ} \mathrm{C}$ peak with Dy concentration. There is a steady increase in the sensitivity up to $0.5 \mathrm{~mol} \% \mathrm{Dy}$, and thereafter, it decreases slightly. Therefore, $0.5 \mathrm{~mol} \%$ Dy in $\beta$-TCP could be taken as the optimum dopant concentration for maximum output.

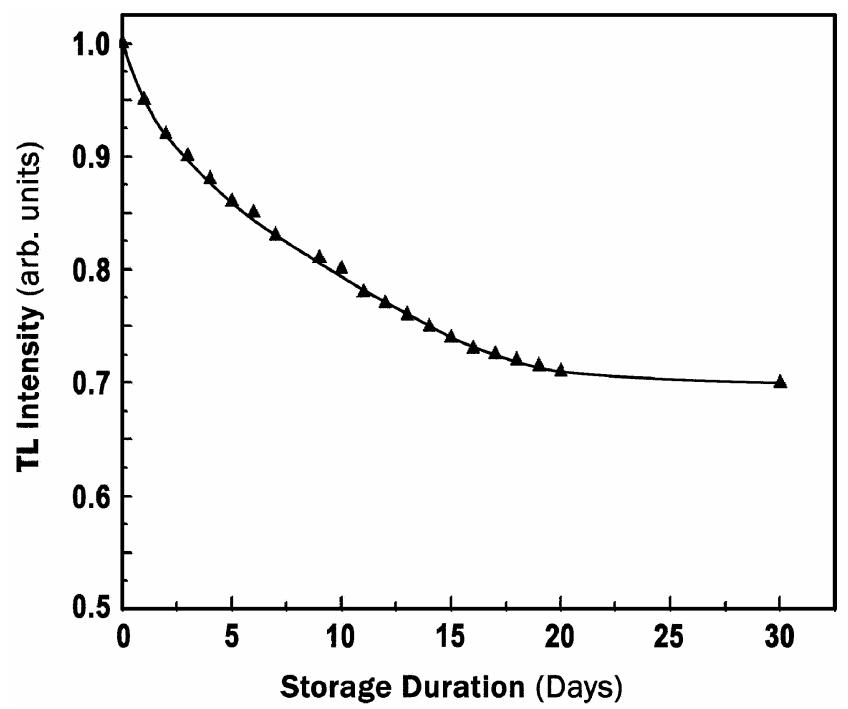

Figure 10. Effect of storage on TL response of X-irradiated $\beta$-TCP : Dy. 


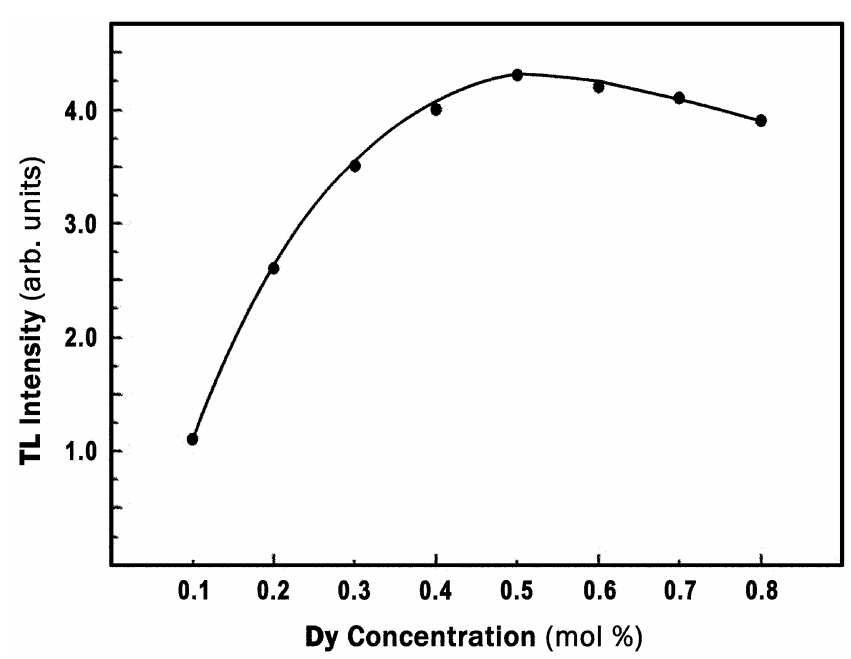

Figure 11. Effect of Dy concentration on the TL response of $220^{\circ} \mathrm{C}$ peak of $\beta$-TCP.

\section{Conclusions}

The $\alpha$ and $\beta$ phases of tricalcium phosphate (TCP) were successfully synthesized through wet precipitation and high temperature solid state routes and doped with Dy and $\mathrm{Eu}$.

Luminescence properties (both PL and TL) of TCP are very sensitive to its crystal phase $(\alpha / \beta)$. The PL study identifies $\mathrm{Dy}^{3+}$ and $\mathrm{Eu}^{3+}$ ions as the emission centres in the doped TCP matrix.

Though the TL efficiency of $\alpha$-TCP is more compared to $\beta$-TCP, the former is less suited for TL applications as its peak temperature is on the lower side, which indicates high fading rate.

An interesting outcome of the TL studies on doped tricalcium phosphate is that the phase transition from $\beta$ to $\alpha$ during annealing could easily be identified through emission parameters.

Dysprosium is found to be an efficient dopant in $\beta$ TCP matrix compared to europium for maximum TL efficiency. However, the fading rate of $\beta$-TCP : Dy is found to be higher than the conventionally suggested response.

\section{Acknowledgements}

The authors are thankful to Dr K S V Nambi, Former HOD, Environmental Assessment Division, Bhabha Atomic Research Centre, Mumbai, whose suggestions helped in conceiving this work. The authors thank $\mathrm{Dr}$ M T Jose, Radiological Safety Division, Indira Gandhi Centre for Atomic Research, Kalpakkam, for his valuable suggestions during the course of this work. Thanks are also due to Dr K Rajendra Babu and other staff of the Department of Physics, Mahatma Gandhi College, Thiruvananthapuram, for their kind support. The authors express their gratitude to Mr S Vijayan and Mr S Sureshbabu, Bioceramics Lab, Sree Chitra Tirunal Institute for Medical Sciences and Technology, Thiruvananthapuram, for their technical help during the course of this work. The financial assistance provided by KSCSTE, Government of Kerala, for this work is gratefully acknowledged.

\section{References}

Band A M, Dhoble S J, Pode R B and Deshmukh B T 1997 Proc. nat. conf. on luminescence and its applications (New Delhi: Allied Publishers) p. 157

Dhabekar B S, Sanayee S S, Shinde S S and Bhatt B C 2002 Proc. of nat. seminar on luminescence and its applications, NSLA 9 (Baroda: Luminescence Society of India) p. 70

Doata A, Pelleb F and Lebuglea A 2005 J. Solid State Chem. 1782354

Ducheyne P and de Groot K 1981 J. Biomed. Mater. Res. 15 441

Fukuda Y, Ohtaki H, Taniguchi S and Takeuchi N $1992 \mathrm{~J}$. Mater. Sci. Lett. 11731

Fukuda Y, Ohtaki H, Tomita A and Takeuchi N 1993 Radiat. Prot. Dosim. 47201

Jarcho M, Bolen C H, Thomas M B, Bobick J, Kay J F and Doremus R H 1976 J. Mater. Sci. 112027

Kanazawa T 1989 Inorganic phosphate materials, in Materials science monographs (Amsterdam: Elsevier) 52

Kottaiswamy M, Mohan Rao M and Jayakumar D 1997 Proc. nat. conf. on luminescence and its applications (New Delhi: Allied Publishers) p. 153

Mckeever S W S 1985 Thermoluminescence of solids (Cambridge: Cambridge University Press)

McKinlay A F 1981 Thermoluminescence dosimetry (Medical physics handbook) (New York: Taylor and Francis)

Michel P I, Guilhot J B and Huguen D 2001 Opt. Mater. 17 409

Mizuguchi K and Fukuda Y 1999 Radiat. Prot. Dosim. 84301

Nakashima K, Takamib M, Ohtac M, Yasued T and Yamauchie J 2005 J. Lumin. 111113

Ohtaki H, Fukuda Y and Takeuchi N 1993 Radiat. Prot. Dosim. 47119

Pradhan A S 1997 Proc. nat. conf. on luminescence and its applications (New Delhi: Allied Publishers) p. 83

Sanayee S S, Shirva V K, Bhatt B C, Shinde S S, Naranji S M and Moharil S V 1997 Proc. nat. conf. on luminescence and its applications (New Delhi: Allied Publishers) p. 219

Schipper W J, Hamelink J J, Langeveld E M and Blasse G 1993 Phys. D: Appl. Phys. 261487

Seshagiri T K, Natarajan V and Sastry M D 1992 Thermoluminescence and its applications (eds) K V R Murthy et al (New Delhi: Tata McGraw-Hill)

Welker T 1991 J. Lumin. 48-49 49 